\title{
HEMODYNAMIC CHANGES DURING THIOPENTAL ANESTHESIA \\ IN HUMANS : CARDIAC OUTPUT, STROKE VOLUME, TOTAL PERIPHERAL RESISTANCE, AND INTRATHORACIC BLOOD VOLUME ${ }^{1,2}$
}

\author{
By BENJAMIN ETSTEN AND T. H. LI 3 \\ (From the Department of Anesthesia, Tufts College Medical School and New England Center \\ Hospital, Boston, Mass.)
}

(Submitted for publication August 13, 1954; accepted November 24, 1954)

Thiopental, monosodium 5-ethyl-5-(1-methylbutyl)-thiobarbiturate, is extensively used in clinical anesthesia either as a primary anesthetic agent for short surgical procedures or as a supplementary drug in conjunction with other anesthetics. Fatalities have been attributed to its use $(1,2)$ and cardiac arrest has occurred following its administration to patients under spinal anesthesia (3).

Most of the information concerning the cardiocirculatory effects of thiopental has been derived from animal experimentation $(4,5)$. Although there have been numerous clinical reports describing changes in blood pressure, pulse rate and respiration during its administration, quantitative investigations on the effect of this agent on the cardiocirculatory dynamics in man are meagre $(6,7)$. In view of its extensive use and the complications ascribed to its administration, a critical analysis of the hemodynamic changes which occur at various levels of thiopental narcosis in patients prior to surgery will form the basis of this communication.

\section{METHOD}

Cardiac output and related hemodynamic determinations were obtained on 14 patients prior to surgery. These subjects were in good physical condition and had no discernible cardiovascular or pulmonary disease (average age- -41 years; range 14 to 60 years). The preanesthetic dosage of morphine and scopolamine varied according to the age, weight, and physical status. Ninety minutes after a subcutaneous administration of morphine (5.4 to $8.0 \mathrm{mgm}$.) and scopolamine (0.26 to $0.43 \mathrm{mgm}$.),

1 Aided by a research grant from the National Institutes of Health, U. S. Public Health Service, No. H-1711 (C2).

2 A preliminary report was presented at the meeting of the Federation of American Societies for Experimental Biology, Atlantic City, New Jersey, April 16, 1954.

${ }^{3}$ Charlton Fellow-Tufts College Medical School. the patient was brought to a quiet anesthesia preparation room for study and was placed in the supine position with one arm resting on a pillow above the angle of Louis. A 15-gauge needle was placed in the median basilic vein of the elevated arm and was connected with a three-way stopcock for the administration of T-1824 (Evans Blue) and thiopental. A thin-walled 18-gauge needle with stylet was inserted into the brachial artery of the other arm for the collection of blood samples. Electrocardiograph electrodes were appropriately placed and electroencephalograph electrodes were positioned according to the method of Kiersey, Bickford, and Faulconer (8).

Control observations were made after a steady resting state was maintained for at least $\mathbf{3 0}$ minutes. It was considered that a basal resting state was obtained only when the pulse rate was below 84 per minute. An intravenous drip of 0.2 per cent thiopental was administered slowly. The rate of flow was adjusted to produce specific levels of anesthesia as defined by Kiersey, Bickford, and Faulconer (8) and monitored by continuous EEG tracings. The observations were correlated according to the specific levels of narcosis as shown in the following classification:

$\begin{array}{cc}\text { E.E.G. Pattern } & \text { Level of Anesthesia } \\ \text { O } & \text { Control } \\ \text { I } & \text { Hypnosis } \\ \text { II } & \text { Light } \\ \text { III and IV } & \text { Deep }\end{array}$

The average duration of each study was two hours and the total dosage of thiopental ranged from 0.6 to $1.0 \mathrm{gm}$.; 100 per cent oxygen was administered throughout the procedure.

After specific levels of thiopental anesthesia were maintained for 15 minutes, the following observations were made: cardiac output, mean arterial pressure, total peripheral resistance, stroke volume, mean circulation time, intrathoracic blood volume, arterial oxygen content and capacity, carbon dioxide content and $\mathrm{pH}$, and electrocardiographic recording.

The cardiac output was determined by the dye dilution technique (9). The injection of T-1824 in a 0.5 per cent solution and collection of samples from the brachial artery were carried out according to the modified method of Etsten, Li, and Fisher $(10,11)$. The stand- 
ard deviation of the difference between the Fick and dye methods was 12.7 per cent, and the standard deviation for the difference of duplicate values of cardiac indexes by the modified dye technique was 2.4 per cent $(10,11)$. Brachial arterial pressure was measured by means of a Statham strain gauge (model P-23A) and continuously recorded on a multi-channel, direct-writing oscillograph with a frequency response of 60 C.P.S. The mean arterial pressure was determined by planimetry of the pulse wave. Samples of arterial blood were drawn immediately after the cardiac output determination for analysis of oxygen content and capacity, carbon dioxide content, and $\mathrm{pH}$ (Cambridge Electron Ray $\mathrm{pH}$ Meter-Research Model). Values of the $\mathrm{pH}$ were accepted when the duplicates checked within a 0.02 unit. The carbon dioxide tension was calculated from the carbon dioxide serum content and $\mathrm{pH}$ values using the nomogram of Peters and Van Slyke (12). The hematocrit was determined by centrifuging the heparinized blood in Wintrobe tubes. The following values were calculated from the formulae:

1. Cardiac output: The cardiac output was calculated from the dye dilution curve according to the modified method of Hamilton, Moore, Kinsman, and Spurling $(10,13): F=\frac{60 \times I}{S} \times \frac{100}{100-\mathrm{H}}: \mathrm{F}$ represents the cardiac output in liters per minute, I the amount of injected dye in mgm., $\mathrm{H}$ the hematocrit (not corrected for trapped plasma) and $S$ the sum of all the dye concentrations in the primary circulation taken at 1-second intervals.

A semi-log plot is made from the experimental data and the value of $S$ is obtained in the following manner $(10,11,14): S=A+B$. A equals the sum of $C_{1}+C_{2}+$ ... $C_{x}$, i.e., all dye concentrations in mgm. per liter except the last term on the descending limb of the primary curve before recirculation starts. This last term is designated $C_{x+1}$, and $C_{\mathbb{x}}$ is the one just prior to this last term. A straight line is extrapolated from $C_{\mathbb{k}}$, and $B$ equals the sum of the concentrations obtained from the extrapolated portion of the descending limb of the curve. The last three or more points of the primary circulation fall on a straight line corresponding to an exponential fall in dye concentration. From these few points a straight line is extrapolated to complete the graph of the primary circulation. The sum of the dye concentrations in this extrapolated portion of the graph is computed by the formula for geometric series. $B=\frac{C_{K+1}}{1-r}$ and is added to the sum of $A ; r$ is the ratio of any term of the series to the preceding term.

2. Mean circulation time: The mean circulation time is the time taken by the dye particle of average speed to pass through the veins from the point of injection, the right heart, the lungs, the left heart, and the arteries to the point of sampling. It is calculated from the formula:

$$
\text { M.C.T. }=\frac{C_{1} T_{1}+C_{2} T_{2}+\cdots C_{K} T_{K}+\frac{C_{K+1}}{1-r}\left(T_{K+1}+\frac{r}{1-r}\right)}{S}
$$

The T's represent the lengths of time between injection and the sample at each second throughout the primary curve until recirculation appears. The last term $\frac{C_{K+1}}{1-r}\left(T_{K+1}+\frac{r}{1-r}\right)$ equals the sum of the products of all extrapolated terms multiplied by time.

The mean circulation time was determined by plotting on semi-logarithmic graph paper the concentration of the dye in each sample against the time of collection of the sample. The mean circulation time is in close agreement to a vertical line that divides the area subtended by the primary dye curve into two equal parts.

3. Intrathoracic blood volume $(15,16)$ : The volume calculated from the mean circulation time $\times \frac{\mathrm{C} . \mathrm{O} \text {. }}{60}$ represents the volume of circulating blood from the point of injection of the dye in the peripheral arm veins, right heart, pulmonary vasculature, the left heart and the arteries to the point of sampling.

Intrathoracic blood volume (liters) $=\frac{\text { C.O. }}{60} \times$ M.C.T., where C.O. = cardiac output in L. per min. and M.C.T. = mean circulation time in seconds. Changes of the intrathoracic blood volume were not considered as significant when they were less than 15 per cent of the control values $(7,17)$.

4. Total peripheral resistance:

$$
\begin{aligned}
& \text { T.P.R. }\left(\frac{\text { dynes }- \text { sec. }}{\mathrm{cm} .^{5}}\right) \\
& =\frac{\text { Mean arterial blood pres. }(\mathrm{mm} . \mathrm{Hg}) \times 1,332}{\text { C.O. (cc./sec.) }}
\end{aligned}
$$

The gradient was represented by the arterial pressure only, since it has been found that the right auricular pressure is unchanged during thiopental anesthesia (18).

\section{Left ventricular work:}

L.V.W (Kg.-meters per min.) $=0.0135 \times$ C.O. (L. per min.) $\times$ mean arterial blood pressure $(\mathrm{mm} . \mathrm{Hg}$ )

6. Statistical analysis: The per cent change refers to the mean per cent change of each group. Each patient served as his own control. Only the control values for those patients studied under a specific level of anesthesia were used to calculate the per cent change found during that specific level. The mean of the control values for the whole group was not used to calculate the per cent change found during any specific level of anesthesia since some patients were not studied throughout all of the levels.

The probability or "P" value was obtained by the method of the Fisher's T-test.

\section{RESULTS}

The pertinent data are summarized in Table I; the mean, the standard deviation, and the standard error of the mean of the absolute values are shown in Table II; and the average percentage changes during the different levels of thiopental anesthesia are shown in Table III.

Cardiac index: The mean cardiac index during the resting state was $3.37 \mathrm{~L}$. per min. per $\mathrm{M}^{2}$ and 
TABLE I

Physical characteristics, hemodynamics, and arterial blood gas data before and during thiopental anesthesia in 14 patients prior to surgery

\begin{tabular}{|c|c|c|c|c|c|c|c|c|c|c|c|c|c|c|c|c|c|}
\hline Pationt & sex & Age & B.s.A. & E.E.G. & $\begin{array}{l}\text { Pulse } \\
\text { Rate }\end{array}$ & $\begin{array}{l}\text { Moan } \\
\text { Art. } \\
\text { B.P. }\end{array}$ & c.o. & c.1. & $\begin{array}{l}\text { Stroke } \\
\text { Voluse }\end{array}$ & T.P.R. & L.v.v. & n.c.т. & I.B.v.1. & Het. & $\mathrm{O}_{2}^{\text {Art }}$ sit. & $\mathrm{PCO}_{2}$ & pn \\
\hline - & & Yrs. & $n^{2}$ & Level & $\frac{\text { Bents }}{\text { PIn. }}$ & = $\mathrm{Hz}$ & L/min & $L / \ln n / n^{2}$ & .c.c. & Dynes-s & $\frac{\mathrm{Kg} \text {-meters }}{\text { Tin. }}$ & sec. & $\underline{L / m^{2}}$ & $\underline{x}$ & $\underline{s}$ & $=\mathrm{H}_{\mathrm{n}}$ & $\underline{37^{\circ} \mathrm{C}}$ \\
\hline 1. R.L. & $\mathbf{r}$ & 59 & 1.64 & \begin{tabular}{|c|} 
Control \\
$1-2$ \\
$1-2$ \\
\end{tabular} & $\begin{array}{l}60 \\
60 \\
72 \\
\end{array}$ & $\begin{array}{l}76 \\
79 \\
77 \\
\end{array}$ & $\begin{array}{l}5.92 \\
6.11 \\
6.20 \\
\end{array}$ & $\begin{array}{l}3.61 \\
3.73 \\
3.78 \\
\end{array}$ & $\begin{array}{r}98 \\
102 \\
86 \\
\end{array}$ & $\begin{array}{r}1030 \\
1050 \\
990 \\
\end{array}$ & $\begin{array}{l}6.0 \\
6.6 \\
6.5 \\
\end{array}$ & \begin{tabular}{l|}
23.0 \\
19.7 \\
20.8 \\
\end{tabular} & $\begin{array}{l}1.38 \\
1.24 \\
1.33 \\
\end{array}$ & \begin{tabular}{l|}
38 \\
37 \\
37 \\
\end{tabular} & $\begin{array}{l}-- \\
-- \\
\end{array}$ & $\begin{array}{l}\because- \\
\because \\
\end{array}$ & $\because$ \\
\hline 2. B.X. & $x$ & 30 & 1.75 & $\begin{array}{c}\text { Control } \\
1-2 \\
3-5 \\
\end{array}$ & $\begin{array}{l}78 \\
78 \\
90 \\
\end{array}$ & $\begin{array}{l}93 \\
79 \\
78 \\
\end{array}$ & $\begin{array}{l}5.26 \\
4.52 \\
4.67 \\
\end{array}$ & $\begin{array}{l}3.01 \\
2.58 \\
2.65\end{array}$ & $\begin{array}{l}67 \\
58 \\
52 \\
\end{array}$ & $\begin{array}{l}1410 \\
1400 \\
1330\end{array}$ & $\begin{array}{l}6.6 \\
4.8 \\
4.9\end{array}$ & \begin{tabular}{l|}
18.1 \\
19.1 \\
18.0
\end{tabular} & $\begin{array}{l}0.91 \\
0.82 \\
0.79\end{array}$ & $\begin{array}{l}42 \\
41 \\
39\end{array}$ & $\ddot{9 i}$ & $\begin{array}{l}-15 \\
42\end{array}$ & $\begin{array}{l}7.58 \\
7.22 \\
7.42\end{array}$ \\
\hline 3. A.B. & $\mathbf{r}$ & 49 & 1.53 & $\begin{array}{c}\text { Control } \\
\text { Bypnosis } \\
1-2 \\
3-5 \\
\end{array}$ & $\begin{array}{r}122 \\
108 \\
88 \\
96 \\
\end{array}$ & $\begin{array}{r}111 \\
99 \\
105 \\
102 \\
\end{array}$ & $\begin{array}{l}7.49 \\
5.86 \\
4.43 \\
4.31 \\
\end{array}$ & $\begin{array}{l}4.89 \\
3.83 \\
2.89 \\
2.82 \\
\end{array}$ & $\begin{array}{l}61 \\
54 \\
50 \\
45 \\
\end{array}$ & $\begin{array}{l}1180 \\
1350 \\
1890 \\
1890 \\
\end{array}$ & $\begin{array}{r}11.2 \\
7.8 \\
6.3 \\
5.9 \\
\end{array}$ & $\begin{array}{r}10.2 \\
9.6 \\
12.4 \\
14.9 \\
\end{array}$ & $\begin{array}{l}0.83 \\
0.61 \\
0.60 \\
0.70 \\
\end{array}$ & $\begin{array}{l}41 \\
40 \\
38 \\
37 \\
\end{array}$ & $\begin{array}{r}92 \\
96 \\
100 \\
100 \\
\end{array}$ & $\begin{array}{l}32 \\
34 \\
66 \\
66 \\
\end{array}$ & $\begin{array}{l}7.35 \\
7.34 \\
7.28 \\
7.28 \\
\end{array}$ \\
\hline 4. 1.L. & $\mathbf{r}$ & 36 & 3.58 & $\begin{array}{c}\text { Control } \\
3-5 \\
3-5 \\
3-5 \\
\end{array}$ & $\begin{array}{l}65 \\
63 \\
78 \\
90 \\
\end{array}$ & $\begin{array}{l}75 \\
77 \\
76 \\
78 \\
\end{array}$ & $\begin{array}{l}7.35 \\
5.50 \\
4.58 \\
5.15 \\
\end{array}$ & $\begin{array}{l}4.65 \\
3.48 \\
2.90 \\
3.26 \\
\end{array}$ & $\begin{array}{r}113 \\
87 \\
58 \\
57 \\
\end{array}$ & $\begin{array}{r}820 \\
1120 \\
11330 \\
1210 \\
\end{array}$ & $\begin{array}{l}7.4 \\
5.7 \\
4.7 \\
5.4 \\
\end{array}$ & $\begin{array}{l}16.1 \\
15.4 \\
12.3 \\
15.7 \\
\end{array}$ & $\begin{array}{l}1.25 \\
0.94 \\
0.59 \\
0.86 \\
\end{array}$ & $\begin{array}{l}37 \\
37 \\
36 \\
36 \\
\end{array}$ & $\begin{array}{l}96 \\
106 \\
110 \\
117 \\
\end{array}$ & $\begin{array}{l}47 \\
31 \\
70 \\
57 \\
\end{array}$ & $\begin{array}{l}7.34 \\
7.34 \\
7.15 \\
7.32 \\
\end{array}$ \\
\hline 5. 8.x. & $\mathrm{z}$ & 28 & 1.63 & $\begin{array}{c}\text { Control } \\
1-2 \\
1-2 \\
3-5 \\
\end{array}$ & $\begin{array}{l}79 \\
75 \\
75 \\
85 \\
\end{array}$ & $\begin{array}{l}69 \\
64 \\
70 \\
50 \\
\end{array}$ & $\begin{array}{l}4.61 \\
3.69 \\
4.41 \\
3.52 \\
\end{array}$ & $\begin{array}{l}2.83 \\
2.26 \\
2.71 \\
2.16 \\
\end{array}$ & $\begin{array}{l}61 \\
49 \\
59 \\
41 \\
\end{array}$ & $\begin{array}{l}1200 \\
1400 \\
1270 \\
1140 \\
\end{array}$ & $\begin{array}{l}4.3 \\
3.2 \\
4.2 \\
2.4 \\
\end{array}$ & $\begin{array}{l}20.7 \\
18.0 \\
21.6 \\
18.9 \\
\end{array}$ & $\begin{array}{l}0.97 \\
0.68 \\
0.78 \\
0.85 \\
\end{array}$ & $\begin{array}{l}37 \\
36 \\
35 \\
34 \\
\end{array}$ & \begin{tabular}{r}
96 \\
97 \\
105 \\
\hdashline- \\
\end{tabular} & $\begin{array}{l}46 \\
43 \\
37 \\
-0 \\
\end{array}$ & $\begin{array}{l}7.36 \\
7.30 \\
7.30 \\
-.- \\
\end{array}$ \\
\hline 6. J.s. & * & 60 & 2.81 & $\begin{array}{c}\text { Hypnos is } \\
1-2 \\
3-5 \\
\end{array}$ & $\begin{array}{l}56 \\
70 \\
90 \\
\end{array}$ & $\begin{array}{l}100 \\
112 \\
128 \\
\end{array}$ & $\begin{array}{l}6.26 \\
6.22 \\
5.04 \\
\end{array}$ & $\begin{array}{l}3.45 \\
3.43 \\
2.77 \\
\end{array}$ & $\begin{array}{r}112 \\
89 \\
56 \\
\end{array}$ & $\begin{array}{l}1280 \\
1440 \\
2040 \\
\end{array}$ & $\begin{array}{l}8.5 \\
8.4 \\
8.8 \\
\end{array}$ & $\begin{array}{l}19.5 \\
19.7 \\
19.0 \\
\end{array}$ & $\begin{array}{l}1.12 \\
1.13 \\
0.88 \\
\end{array}$ & $\begin{array}{l}41 \\
40 \\
40 \\
\end{array}$ & $\begin{array}{c}95 \\
100 \\
100 \\
\end{array}$ & $\begin{array}{l}52 \\
58 \\
56 \\
\end{array}$ & $\begin{array}{r}7.32 \\
7.25 \\
7.33 \\
\end{array}$ \\
\hline 7. M.D. & $\mathbf{r}$ & 60 & 1.60 & $\begin{array}{c}\text { Control } \\
1-2\end{array}$ & $\begin{array}{l}78 \\
96\end{array}$ & $\begin{array}{l}107 \\
137\end{array}$ & $\begin{array}{l}4.36 \\
4.09\end{array}$ & $\begin{array}{l}2.71 \\
2.56\end{array}$ & $\begin{array}{l}56 \\
43\end{array}$ & $\begin{array}{l}1960 \\
2680\end{array}$ & $\begin{array}{l}6.3 \\
7.6\end{array}$ & $\begin{array}{l}14.5 \\
14.5\end{array}$ & $\begin{array}{l}0.69 \\
0.62\end{array}$ & $\begin{array}{l}42 \\
41\end{array}$ & $\begin{array}{l}93 \\
92\end{array}$ & $\because$ & $\because$ \\
\hline
\end{tabular}

TABLE I-Continued

\begin{tabular}{|c|c|c|c|c|c|c|c|c|c|c|c|c|c|c|c|c|c|}
\hline Fatsent & $\operatorname{sen}$ & Age & B.s.A. & x.5.G. & $\begin{array}{l}\text { Pulsee } \\
\text { Rate }\end{array}$ & $\begin{array}{l}\text { Eoen } \\
\text { Art. } \\
\text { B.P. }\end{array}$ & c.o. & c.1. & $\begin{array}{l}\text { Stroke } \\
\text { Volues }\end{array}$ & T.P.R. & L.V.ซ. & M.c.t. & I.B.V.I. & Bct. & Art. & $\mathrm{PCO}_{2}$ & pels \\
\hline & & Tre. & $n^{2}$ & Level & 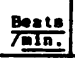 & $=\mathrm{H}_{1}$ & $\underline{w / a 1 n}:$ & wineln/e & c.c. & Dreas-5 & Ex-mer: & eec. & $\underline{w} /^{2}$ & $\underline{\mathbf{z}}$ & $\underline{z}$ & $=8$ & $37^{\circ} \mathrm{C}$ \\
\hline •. c.n. & $\mathbf{n}$ & 56 & 2.88 & $\begin{array}{c}\text { Control } \\
\text { Hypnos is } \\
1-2 \\
1-2\end{array}$ & $\begin{array}{l}60 \\
72 \\
70 \\
87\end{array}$ & $\begin{array}{l}109 \\
118 \\
102 \\
126\end{array}$ & $\begin{array}{l}6.95 \\
7.06 \\
6.05 \\
5.33\end{array}$ & $\begin{array}{l}3.69 \\
3.76 \\
3.22 \\
2.84\end{array}$ & $\begin{array}{r}116 \\
98 \\
86 \\
61\end{array}$ & $\begin{array}{l}1250 \\
1340 \\
1350 \\
1890\end{array}$ & $\begin{array}{r}10.2 \\
11.3 \\
8.3 \\
9.1\end{array}$ & $\begin{array}{l}22.6 \\
22.1 \\
24.8 \\
19.4\end{array}$ & $\begin{array}{l}1.39 \\
1.38 \\
1.33 \\
0.92\end{array}$ & $\begin{array}{l}42 \\
41 \\
41 \\
40\end{array}$ & $\begin{array}{r}94 \\
94 \\
95 \\
103\end{array}$ & $\begin{array}{l}43 \\
-41 \\
43\end{array}$ & $\begin{array}{c}7.40 \\
7.42 \\
7.41\end{array}$ \\
\hline ง. T.C. & $m$ & 24 & 1.77 & $\begin{array}{l}\text { Control } \\
\text { Hypnosis }\end{array}$ & $\begin{array}{l}60 \\
61 \\
\end{array}$ & $\begin{array}{l}106 \\
102 \\
\end{array}$ & $\begin{array}{l}5.25 \\
5.97 \\
\end{array}$ & $\begin{array}{l}2.96 \\
3.37 \\
\end{array}$ & $\begin{array}{l}87 \\
98 \\
\end{array}$ & $\begin{array}{l}1610 \\
1370 \\
\end{array}$ & $\begin{array}{l}7.5 \\
8.2 \\
\end{array}$ & $\begin{array}{l}18.6 \\
20.4\end{array}$ & $\begin{array}{l}0.92 \\
1.14 \\
\end{array}$ & $\begin{array}{l}51 \\
51\end{array}$ & $\begin{array}{l}93 \\
-- \\
\end{array}$ & 41 & \begin{tabular}{|l|}
7.40 \\
7.37 \\
\end{tabular} \\
\hline 10. E.P. & $m$ & 39 & 1.95 & $\begin{array}{l}\text { Control } \\
\text { Hypnosis }\end{array}$ & $\begin{array}{l}60 \\
72\end{array}$ & $\begin{array}{r}103 \\
-103\end{array}$ & $\begin{array}{l}5.13 \\
5.72\end{array}$ & $\begin{array}{l}2.63 \\
2.92\end{array}$ & $\begin{array}{l}85 \\
79\end{array}$ & $\begin{array}{l}1620 \\
1440\end{array}$ & $\begin{array}{l}7.1 \\
7.9\end{array}$ & $\begin{array}{l}21.8 \\
19.4\end{array}$ & $\begin{array}{l}0.95 \\
0.95\end{array}$ & $\begin{array}{l}48 \\
47\end{array}$ & $=$ & $=$ & $=$ \\
\hline 11. J.B. & 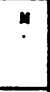 & 30 & 2.92 & $\begin{array}{c}\text { Cont rol } \\
\text { Hypnosis } \\
1-2 \\
3-5 \\
\end{array}$ & $\begin{array}{l}72 \\
62 \\
78 \\
75 \\
\end{array}$ & $\begin{array}{r}96 \\
100 \\
90 \\
72 \\
\end{array}$ & $\begin{array}{l}6.60 \\
6.79 \\
5.18 \\
5.61 \\
\end{array}$ & $\begin{array}{l}3.44 \\
3.53 \\
2.70 \\
2.93\end{array}$ & $\begin{array}{r}92 \\
109 \\
66 \\
75 \\
\end{array}$ & $\begin{array}{l}1160 \\
1180 \\
1390 \\
1030 \\
\end{array}$ & $\begin{array}{l}8.6 \\
9.2 \\
6.3 \\
5.5\end{array}$ & $\begin{array}{l}15.6 \\
18.6 \\
19.4 \\
21.4\end{array}$ & $\begin{array}{l}0.89 \\
1.09 \\
0.86 \\
0.99\end{array}$ & $\begin{array}{l}10 \\
40 \\
38 \\
37\end{array}$ & $=-$ & $\begin{array}{l}= \\
=- \\
=\end{array}$ & $=$ \\
\hline 12. T.D. & m & 24 & 2.00 & $\begin{array}{l}\text { Control } \\
\text { Hypnosis }\end{array}$ & $\begin{array}{l}42 \\
53\end{array}$ & $\begin{array}{l}74 \\
85\end{array}$ & $\begin{array}{l}5.48 \\
5.50\end{array}$ & $\begin{array}{l}2.74 \\
2.75\end{array}$ & $\begin{array}{l}130 \\
104\end{array}$ & $\begin{array}{l}1080 \\
1230\end{array}$ & $\begin{array}{l}5.5 \\
6.3\end{array}$ & $\begin{array}{l}27.9 \\
24.5\end{array}$ & $\begin{array}{l}1.27 \\
1.13\end{array}$ & $\begin{array}{l}46 \\
46\end{array}$ & $=$ & $=$ & $=$ \\
\hline 13. A.F. & $\mathbf{r}$ & 20 & 2.42 & $\begin{array}{c}\text { Control } \\
1-2\end{array}$ & $\begin{array}{r}76 \\
101 \\
\end{array}$ & $\begin{array}{r}107 \\
-- \\
\end{array}$ & $\begin{array}{l}5.06 \\
4.87\end{array}$ & $\begin{array}{l}3.57 \\
3.43\end{array}$ & $\begin{array}{l}67 \\
48\end{array}$ & 1690 & 7.3 & $\begin{array}{l}12.8 \\
12.9\end{array}$ & $\begin{array}{l}0.76 \\
0.74\end{array}$ & $\begin{array}{l}37 \\
36\end{array}$ & $\overline{105}$ & $\overline{47}$ & $7 . \overline{34}$ \\
\hline 14. A.N. & m & 47 & 1.77 & $\begin{array}{l}\text { Control } \\
\text { Hypnosis } \\
3-5\end{array}$ & $\begin{array}{r}70 \\
75 \\
100\end{array}$ & $\begin{array}{l}120 \\
117 \\
130\end{array}$ & $\begin{array}{l}5.42 \\
5.19 \\
4.17\end{array}$ & $\begin{array}{l}3.08 \\
2.95 \\
2.36\end{array}$ & $\begin{array}{l}77 \\
69 \\
42\end{array}$ & $\begin{array}{l}1770 \\
1800 \\
2490\end{array}$ & $\begin{array}{l}8.8 \\
8.2 \\
7.3\end{array}$ & $\begin{array}{l}24.9 \\
20.9 \\
19.6\end{array}$ & $\begin{array}{l}1.27 \\
1.02 \\
0.77\end{array}$ & $\begin{array}{l}45 \\
43 \\
43\end{array}$ & 95 & $\overline{12}$ & $\overline{7.41}$ \\
\hline
\end{tabular}

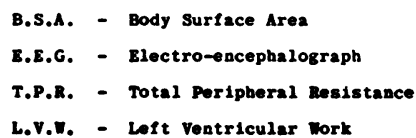

L.V.ซ. - Left Ventricular work

the standard deviation of the sample was \pm 0.72 L. per min. per $\mathbf{M}^{2}$. This value compares favorably with those reported by other investigators (Table IV). The cardiac index did not change significantly during the state of thiopental hypnosis $(P>0.9)(10)$. The state of hypnosis

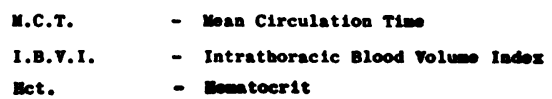

was characterized by the typical EEG barbiturate sleep pattern. The patient was somnolent but would respond by awakening to a strong extraneous stimulus.

The mean cardiac index during light surgical anesthesia was 3.01 L. per min. per M $^{2}$ (S.D. \pm 
TABLE II

Average absolute values during thiopental anesthesia

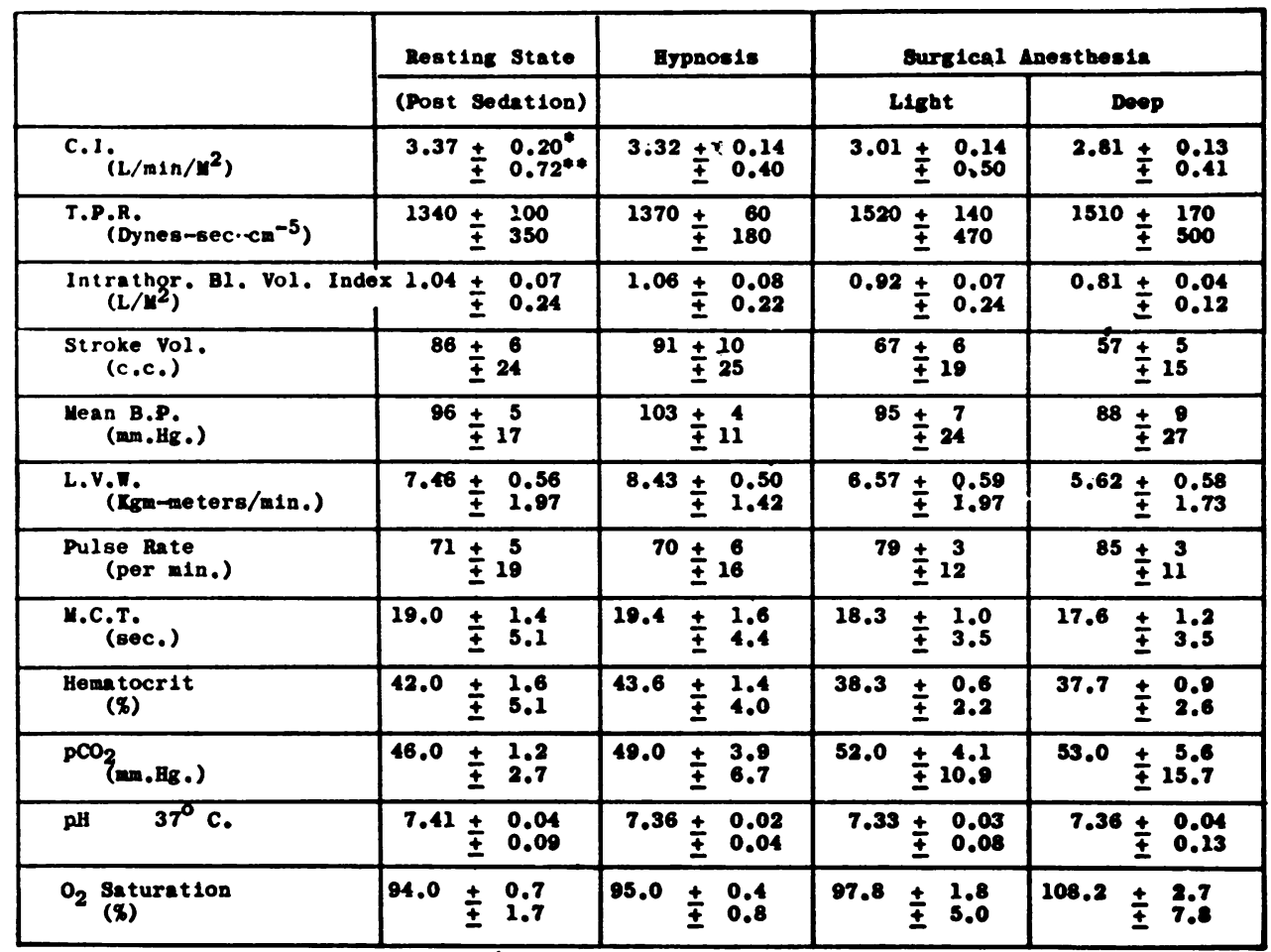

* Standard Frror of the than

* Standard Deviation

$0.50)$, and during deep surgical anesthesia was 2.81 L. per min. per $\mathrm{M}^{2}$ with a standard deviation of $\pm 0.41 \mathrm{~L}$. per min. per $\mathrm{M}^{2}$, as compared to the mean value during the resting state of $3.37 \mathrm{~L}$. per min. per $\mathrm{M}^{2}$ with a standard deviation of \pm 0.72 L. per min. per $\mathbf{M}^{2}$ (Table II). The average decrease of the cardiac index during the light level of anesthesia was 12 per cent $(P>0.3)$ and during the deep level of surgical anesthesia was 25.4 per cent $(P<0.001)$ (Figure 1$)$. Six of the 12 cardiac output values during light surgical anesthesia were reduced 12 per cent below the resting state, and all of the 9 determinations during the deep surgical anesthesia fell below the 12 per cent level (range : 12 to 42 per cent).

Intrathoracic blood volume: The mean intrathoracic blood volume index during the resting state was $1.038 \mathrm{~L}$. per min. per $\mathrm{M}^{2}$ with a standard deviation of $\pm 0.238 \mathrm{~L}$. per min. per $\mathrm{M}^{2}$. It was not significantly changed during the state of hypnosis. Nine of the 12 determinations of the values of the intrathoracic blood volume during the light surgical anesthesia were not significantly changed. The mean value was 12.5 per cent $(\mathrm{P}<0.05)$ below the resting value. Eight of the nine determinations during the deep level fell below 13 per cent. The mean value was reduced 23 per cent $(P<0.001)$ below the control value and the range of reduction was 13 to 52 per cent.

Stroke volume: The mean value dropped from $86 \mathrm{cc}$. (resting state) to $66.5 \mathrm{cc} . \pm 19.4$ (S.D.) at the light level and 57.1 cc. \pm 15.4 (S.D.) at the deep level of anesthesia. Analysis of the percentage changes during the anesthesia levels compared with the resting state showed that the stroke volume was reduced 20 per cent during light anesthesia $(P<0.01)$ and 35 per cent during deep anesthesia $(P<0.001)$. Nine of the 11 determinations of the values of the stroke volume during light surgical anesthesia were reduced 13 to 47 per cent and the values during the deep level ranged from 19 to 50 per cent below the resting state.

Total peripheral resistance: The calculated mean total peripheral resistance during the resting state was 1,340 dynes-sec.-cm..$^{-5}$ with a standard devia- 
BEN JAMIN ETSTEN AND T. H. LI

TABLE III

Hemodynamic changes: thiopental *

\begin{tabular}{|c|c|c|c|}
\hline & \multicolumn{2}{|c|}{ \% Change } & \\
\hline & \multirow[t]{2}{*}{ Hypnos is } & \multicolumn{2}{|c|}{ Surgical Anesthesia } \\
\hline & & Light & Deep \\
\hline C.1. $\mathrm{L} / \mathrm{min} / \mathrm{m}^{2}$ & $+0.5 \%$ & $-11.6 \%$ & $-25.4 \%$ \\
\hline $\begin{array}{l}\text { T.P.R. } \\
\text { Dynes-sec . }-\mathrm{cm}^{-5}\end{array}$ & $+1.8 \%$ & $+18.7 \%$ & $+31.7 \%$ \\
\hline$\underset{\text { Index }}{\text { Intrathor. }} \mathrm{Bl} \underset{\mathrm{L} / \mathrm{m}^{2}}{\mathrm{Vol}}$ & $-1.8 \%$ & $-12.5 \%$ & $-23.0 \%$ \\
\hline $\begin{array}{c}\text { Stroke Volume } \\
\text {.c.c. }\end{array}$ & $-4.8 \%$ & $-19.8 \%$ & $-35.2 \%$ \\
\hline $\begin{array}{l}\text { Mean B.P. } \\
\text { mm. Hg }\end{array}$ & $+1.5 \%$ & $+2.1 \%$ & $-3.6 \%$ \\
\hline L.V. igm.-meters/min. $_{\text {igm }}$ & $+2.3 \%$ & $-9.6 \%$ & $-28.1 \%$ \\
\hline $\begin{array}{c}\text { Pulse Rate } \\
\text { per min. }\end{array}$ & $+7.1 \%$ & $+11.9 \%$ & $+18.8 \%$ \\
\hline $\begin{array}{l}\text { M.C.T. } \\
\text { sec. }\end{array}$ & $-2.7 \%$ & $+0.3 \%$ & $+3.7 \%$ \\
\hline
\end{tabular}

* The mean percentage change of each group includes only those patients who were studied during a specific level of anesthesia. The individual per cent change calculated for a specific level of anesthesia refers to the resting values of the same individual and not to the mean of the control values for the entire group.

TABLE IV

Cardiac index: resting state

\begin{tabular}{|c|c|c|c|c|}
\hline \multirow{2}{*}{ Method } & \multirow{2}{*}{ Author } & \multirow{2}{*}{ Number } & \multicolumn{2}{|c|}{ C.I. $\mathrm{I} / \mathrm{min} / \mathrm{H}^{2}$} \\
\hline & & & Mean \pm S.E. & S.D. \\
\hline Fick & $\begin{array}{l}\text { Stead et al, } 1945 \text { (19) } \\
\text { Cournand et al, } 1945 \text { (20) } \\
\text { Sancetta et al, } 1952 \text { (21) } \\
\text { Doyle et al, } 1953 \text { (22) }\end{array}$ & $\begin{array}{l}18 \\
30 \\
10 \\
53\end{array}$ & $\begin{array}{l}3.37 \\
3.31 \\
3.37 \\
3.50\end{array}$ & $\begin{array}{l} \pm 0.57 \\
\pm 0.54 \\
\pm 0.30\end{array}$ \\
\hline Dye & $\begin{array}{l}\text { Doyle et al, } 1953(22) \\
\text { Etsten and } \mathrm{L1}, 1954(10,21)\end{array}$ & $\begin{array}{l}\left\{\begin{array}{l}53 \\
10\end{array}\right. \\
\left\{\begin{array}{l}47 \\
10^{*}\end{array}\right.\end{array}$ & $\begin{array}{l}3.20 \\
3.20 \pm 0.23 \\
3.29 \pm 0.07 \\
3.37 \pm 0.28\end{array}$ & $\begin{array}{l} \pm 0.20 \\
\pm 0.70 \\
\pm 0.49 \\
\pm 0.58\end{array}$ \\
\hline
\end{tabular}

* Thiopental hypnosis 
CARDIAC INDEX: THIOPENTAL

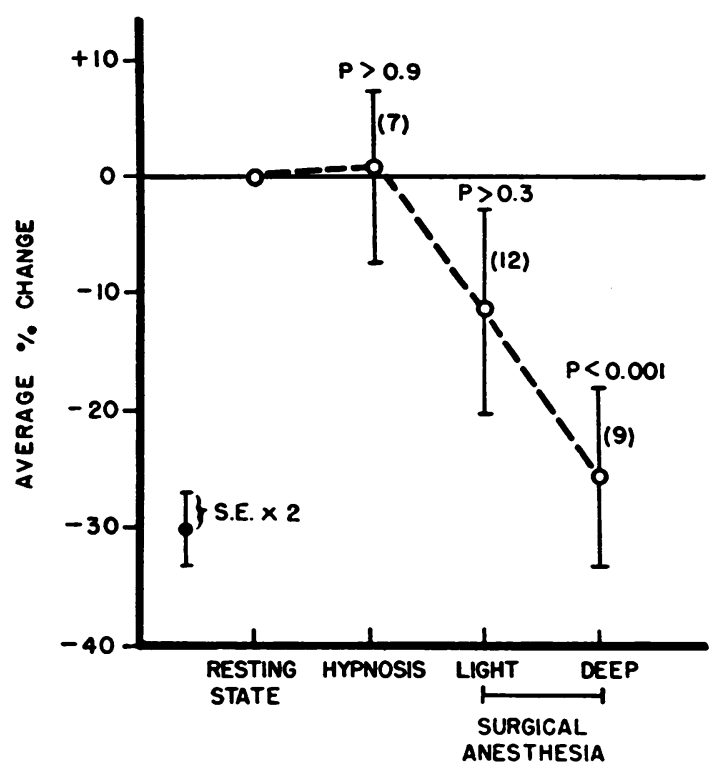

Figure 1

tion of \pm 350 . During the state of hypnosis the mean value was 1,370 dynes-sec.-cm.-5 with a standard deviation of $\pm 180(\mathrm{P}>0.6)$. During light surgical anesthesia the total peripheral resistance increased 19 per cent $(P<0.01)$ and during deep anesthesia 32 per cent $(\mathrm{P}<0.01)$ (Tables II and III).

THIOPENTAL HEMODYNAMIC CHANGES

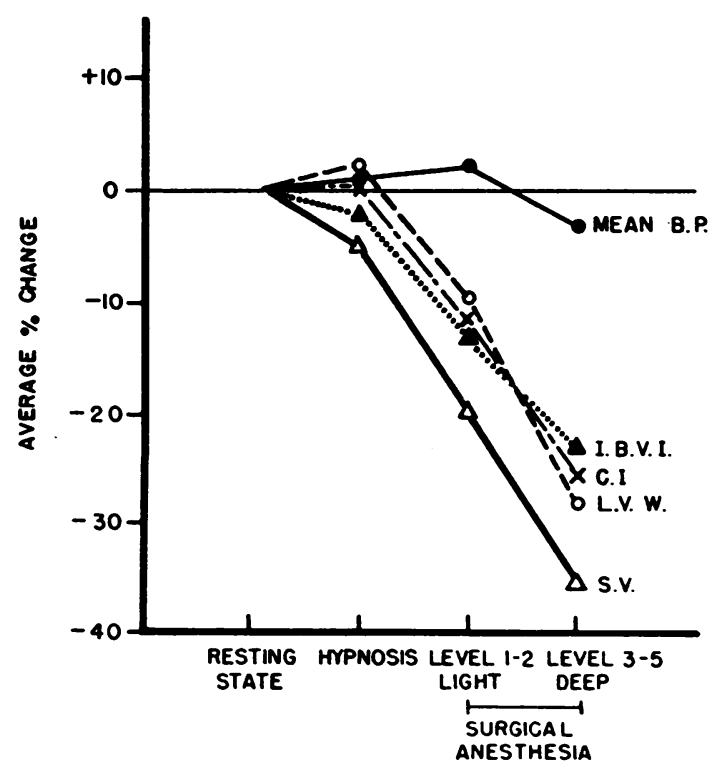

FIGURE 2
Mean arterial blood pressure: The mean arterial blood pressure was $96 \mathrm{~mm}$. $\mathrm{Hg}$ with a standard deviation of $\pm 16.9 \mathrm{~mm}$. Hg during the resting state; it did not change significantly during any level of surgical anesthesia $(P>0.9)$ (Tables II and III).

Pulse rate: The pulse rate during the resting state ranged from 60 to 84 with a mean value of 71 per minute (S.D. \pm 19 ). The mean pulse rate was 70 per minute with a standard deviation of $\pm 16(\mathrm{P}>0.6)$ during hypnosis, 79 per minute $(\mathrm{S} . \mathrm{D} . \pm 12)(\mathrm{P}>0.05)$ during light anesthesia, and 85 per minute (S.D. \pm 11$)(P<$ 0.01) during deep anesthesia (Table II). The average increase of pulse rate during hypnosis was 7.1 per cent (seven determinations) $(\mathrm{P}>$ $0.5)$; during light anesthesia the increase was 11.9 per cent (twelve determinations) $(\mathrm{P}>0.05)$. Both changes were not statistically significant. During deep surgical anesthesia, however, the

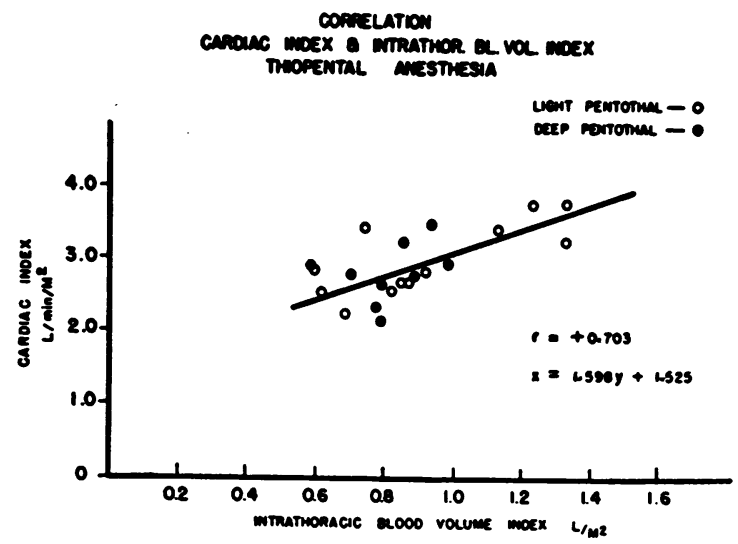

FIG. 3 o

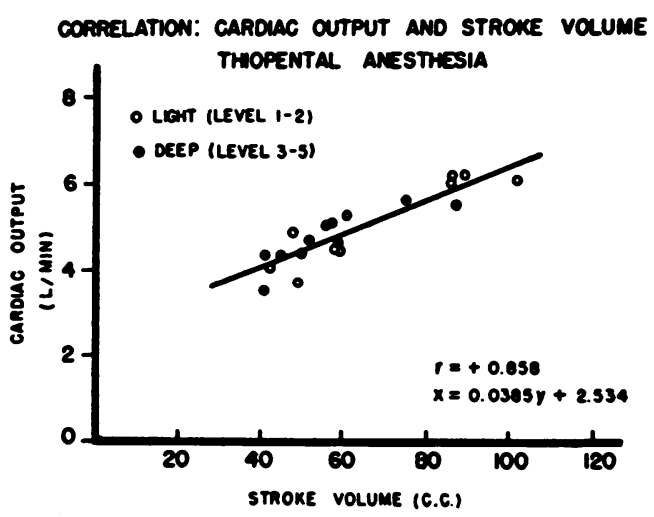

FIG. 3 b

Figure 3 
average increase (nine determinations) was 18.8 per cent $(P<0.02)$.

Left ventricular work: The left ventricular work ranged from 4.3 to $11.2 \mathrm{Kg}$--meters per minute with a mean value of $7.46 \mathrm{Kg}$--meters per minute. During hypnosis the average change was an increase of 2.3 per cent $(P>0.5)$. During light surgical anesthesia, the left ventricular work decreased 9.6 per cent $(P>0.1)$ and during deep anesthesia the left ventricular work was reduced 28 per cent (S.D. \pm 15 per cent $)(P<0.001)$ (Tables II and III).

Mean circulation time: The mean circulation time was 19 seconds with a standard deviation of \pm 5 seconds during the resting state. There was no significant change in the mean circulation time during the three levels of thiopental anesthesia (Table II), the average changes ranging from -2.7 to +3.7 per cent.

Oxygen saturation: The oxygen saturation ranged from 92 to 96 per cent during the resting state and ranged from 95 to 108 per cent throughout all the levels of surgical anesthesia (Tables I and II).

Carbon dioxide tension: The mean $\mathrm{pCO}_{2}$ during the resting state was $46 \mathrm{~mm}$. $\mathrm{Hg}$ with a standard deviation of $\pm 2.74 \mathrm{~mm}$. Hg. During hypnosis the average $\mathrm{pCO}_{2}$ value was $49 \mathrm{~mm}$. $\mathrm{Hg}$; during light anesthesia was $52 \mathrm{~mm}$. $\mathrm{Hg}$; and during deep surgical anesthesia was $53 \mathrm{~mm}$. $\mathrm{Hg}$

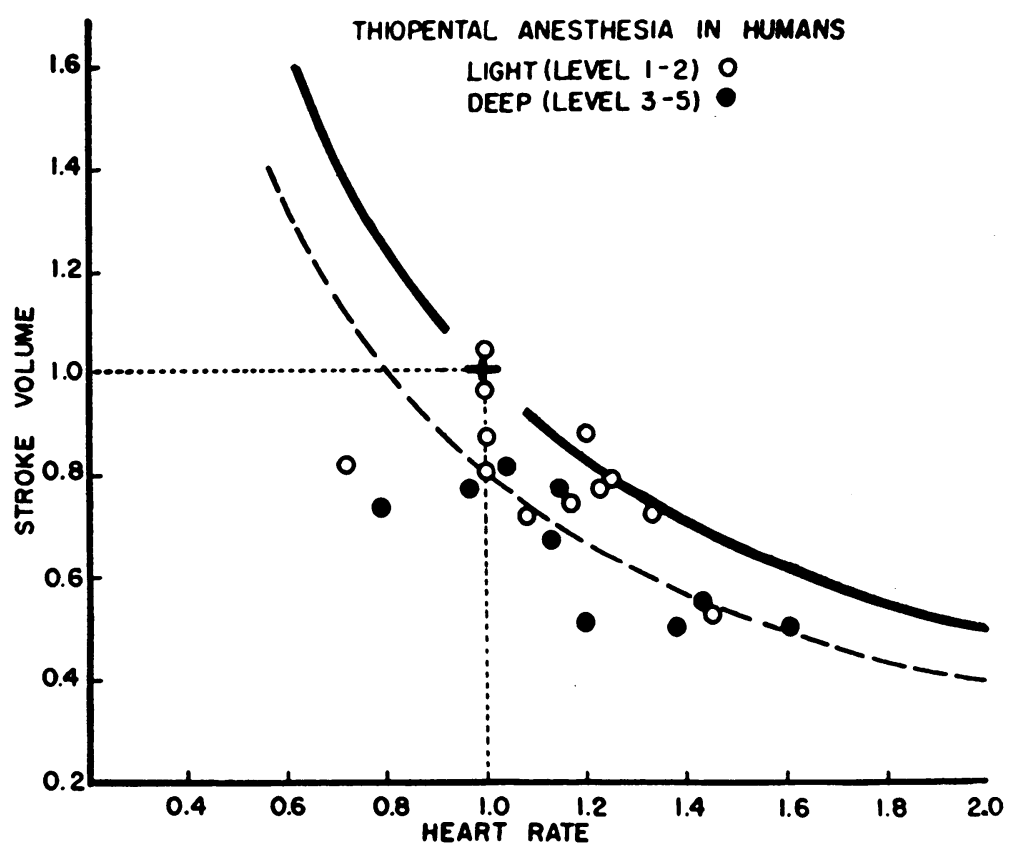

Fig. 4. The Effect of Thiopental Anesthesia on the Stroke Volume AND HEART RATE

Each point represents a departure from the resting state, indicating the value of the stroke volume and the heart rate at different levels of thiopental anesthesia. Heart rate and stroke volume are expressed each as a fraction of the resting value, which is indicated by the cross $(+)$ at the point 1.0, 1.0. All the other points are the experimental values in reference to the resting value. The solid line is a rectangular hyperbola passing through the cross. This solid line consists of points where the product of heart rate and stroke volume, or the cardiac output, is constant and normal. The broken line represents a reduction of the cardiac output, 20 per cent below the resting value. Open circles: light and closed circles: deep thiopental anesthesia. During the light and deep thiopental anesthesia the stroke volume was decreased and heart rate increased with the resultant cardiac output values scattering more or less uniformly around the broken line, i.e., 20 per cent below the resting value. 
(Tables I and II). These values ranged from 31 to 66 (seven determinations during light and eight determinations during deep surgical anesthesia). In three experiments there was a slight increase in $\mathrm{pCO}_{2}$. In all the other experiments there was either no change or even a slight decrease. All the variations of the $\mathrm{pCO}_{2}$ during thiopental anesthesia were statistically insignificant $(\mathrm{P}>0.1)$.

Correlation of cardiac index, intrathoracic blood volume, and stroke volume: Since the cardiac index, intrathoracic blood volume index, and stroke volume were all reduced (Figure 2) during surgical anesthesia, it was deemed worthwhile to determine the correlation coefficient among these three variables. The correlation coefficient between the cardiac index and intrathoracic blood volume index was +0.703 and between cardiac output and stroke volume was \pm 0.858 (Figure 3, $a$ and $b$ ). There was also a positive correlation between the intrathoracic blood volume and stroke volume $(r=+0.765)$.

Relationship of the stroke volume and pulse rate to the cardiac output during thiopental anesthesia: The usual inverse relationship between pulse rate and stroke volume was not well maintained during thiopental anesthesia. Although the mean increase of the pulse rate was 18 per cent, the change was not sufficient to compensate for the lowered stroke volume. This is shown in Figure 4.

\section{DISCUSSION}

The hemodynamic changes observed in patients during thiopental anesthesia varied with the levels of narcosis. The cardiac output, stroke volume, and intrathoracic blood volume were progressively reduced as the level of anesthesia deepened (23). Since thiopental was given slowly in low concentration ( 0.2 per cent), respiratory depression and obstruction were avoided. Marked variations of respiratory exchange (24), therefore, can be excluded as a factor in causing lowered cardiac output and lowered intrathoracic blood volume or in concealing changes in the effective auricular filling pressure. Since the central venous and left auricular pressures $(18,25)$ are not elevated during thiopental anesthesia, it is reasonable to state that the decrease in cardiac output is not the result of depression of the heart as a pump.
The effect of thiopental on metabolism may be a possible cause for the decrease in cardiac output. Total body oxygen consumption is lowered by 15 per cent (26), and cerebral metabolism is decreased during thiopental anesthesia $(27,28)$. The lowered requirements of oxygen for tissue metabolism may be partly responsible for the reduction of the cardiac output.

A more likely cause for the lowering of the cardiac output during thiopental anesthesia is a redistribution of the circulatory blood volume. This concept is based on the finding of a positive correlation between the cardiac output, stroke volume, and intrathoracic blood volume (Figure 2).

The intrathoracic blood volume refers to the volume of blood in the basilic vein at the point of insertion of the dye in the heart, lungs and intrathoracic vessels and artery to the point of sampling, frequently designated as the "central blood volume." Since the amount of blood in the major arteries is presumably quite constant $(7,29,30)$ and the source of error related to the variation in volume in the venous system from the point of injection of the dye to the heart is negligible in relation to the volume measured (13), we believe that our measurements of the intrathoracic blood volume mirror changes in the "central pool" of blood which occur during the phases of this study.

It is realized that there are limitations to the method of calculating the intrathoracic blood volume (17). However, Nylin found a close correlation between the heart volume and the type of dilution curves obtained in normal persons. $\mathrm{He}$ also found a close correlation between the results obtained by biplane radiography and the dilution technique (31). The accuracy of the principle employed for the calculation of the "central blood volume" is obviously limited by the accuracy with which the blood flow and the mean circulation time can be measured. Since the mean circulation time remains constant in each subject during all the levels of narcosis, the comparative values of these physiologic volumes are considered as significant.

There are other reports that can be considered in support of this viewpoint. Johnson (7) by means of the dye dilution method found a positive correlation between the lowered cardiac output and central blood volume during spinal and "nar- 
kotal" anesthesia. He also determined the pulmonary blood volume in patients before and during barbiturate anesthesia by injecting dye into the pulmonary artery and collecting blood samples from the brachial artery. Our values for the.intrathoracic blood volume obtained by injecting dye into an antecubital vein showed a similar per cent change during deep thiopental anesthesia. Kopelman and Lee (32) compared the values for the "central pool" obtained by injecting dye into the pulmonary artery in one group of patients and by injecting dye into an antecubital vein in another similar group. They found the same per cent change with each method. Sjöstrand demonstrated a direct relationship of the central blood volume to stroke volume and the cardiac output (29).

It is, therefore, reasonable to postulate that the decrease in intrathoracic blood volume, stroke volume, and cardiac output is caused by a redistribution and pooling of blood in the peripheral circulation that results in a diminished venous return to the heart, hence the reduced cardiac output. Such pooling could be caused by an increase in total vascular capacity. The progressive decrease in hematocrit characteristic of barbiturate anesthesia is highly suggestive of hemodilution and an increase of total vascular capacity (33-36) (Table II). The blockade of vasoconstrictor reflexes during thiopental anesthesia (25) may also be a contributing factor to the pooling.

Further evidence supporting the concept that the circulating blood volume is redistributed during thiopental or other barbiturate anesthesia appears in the literature. It was reported that blood flow through the mesenteric and femoral vessels was diminished (37); that the spleen was enlarged (33); that cerebral utilization of oxygen was depressed in spite of the maintenance of an adequate blood flow $(27,28,38)$; that vasoconstriction occurred in the liver and kidney associated with a decrease in hepatic and renal blood flow $(39,40)$; that depression of vasomotion and capillary venous output in dogs' omentum was associated with a sluggish venous return of blood as anesthesia deepened $(34,35)$; and that a profound vasodilation occurred in the skin and muscle as found by means of water plethysmography, although the blood pressure was unchanged (36).

On the other hand, Greisheimer and his co- workers (5) reported an increase in cardiac output by means of the cuvette oximeter during thiopental anesthesia in dogs. It is known that the rapid intravenous administration of a 2 to 5 per cent concentration of this drug depresses alveolar ventilation $(24,41)$, resulting in an elevated arterial $\mathrm{pCO}_{2}$. The discrepancy, therefore, between our findings and those of Greisheimer can most likely be explained by the influence of increased arterial $\mathrm{pCO}_{2}$ (41), species difference, and possibly the difference in technique.

In contrast to the findings of a reduction in cardiac output, intrathoracic blood volume, and stroke volume during deep surgical anesthesia, there were no significant changes during thiopental hypnosis. It was found that by means of a slow infusion of a dilute concentration of thiopental ( 0.2 per cent), a steady basal state (hypnosis) could be produced in human subjects for the purpose of investigation. Throughout all the stages of narcosis, the mean arterial pressure was maintained at normal levels (Figure 2, Table III), presumably by the compensatory increase in total peripheral resistance.

\section{SUMMARY AND CONCLUSION}

The changes of the cardiac output and related hemodynamics were evaluated in 14 patients anesthetized with thiopental. The cardiac output was unchanged during the state of hypnosis and reduced 25 per cent (S.D. \pm 10 per cent) during deep surgical anesthesia. The changes in the "intrathoracic blood volume" and stroke volume paralleled the changes of the cardiac output. The reduction in "intrathoracic blood volume" found during surgical anesthesia most likely represents the reduction in the central blood pool. It is believed that the reduction in "intrathoracic blood volume" and stroke volume may be the result of the redistribution and pooling of blood, causing a diminished venous return to the heart.

\section{ACKNOWLEDGMENT}

We would like to express our appreciation to Dr. Robert N. Reynolds of the Department of Anesthesiology and to Dr. Harold F. Rheinlander of the Department of Surgery for their invaluable assistance during part of this investigation; and to Dr. Charles V. Robinson of the Department of Biophysics for his expert advice in the statistical analysis. 


\section{REFERENCES}

1. Halford, F. J., A critique of intravenous anesthesia in war surgery. Anesthesiology, 1943, 4, 67.

2. Heard, K. M., The benefits and hazards of pentothal anesthesia. Anesthesiology, 1944, 5, 448.

3. Stephenson, H. E., Jr., Reid, L. C., and Hinton, J. W., Some common denominators in 1200 cases of cardiac arrest. Ann. Surg., 1953, 137, 731.

4. Tatum, A. L., The present status of the barbiturate problem. Physiol. Rev., 1939, 19, 472.

5. Greisheimer, E. M., Ellis, D. W., Baier, H. N., Ring, G. C., Makarenko, L., and Graziano, J., Cardiac output by cuvette oximeter under thiopental. Am. J. Physiol., 1953, 175, 171.

6. Prime, F. J., and Gray, T. C., The effect of certain anesthetic and relaxant agents on circulatory dynamics. Brit. J. Anaesth., 1952, 24, 101.

7. Johnson, S. R., The effect of some anesthetic agents on the circulation in man with special reference to the significance of pulmonary blood volume for the circulatory regulation. Acta chir. Scandinav., 1951, Supplement 158.

8. Kiersey, D. K., Bickford, R. G., and Faulconer, A., Jr., Electro-encephalographic patterns produced by thiopental sodium during surgical operations: Description and classification. Brit. J. Anaesth., 1951, 23, 141.

9. Hamilton, W. F., Riley, R. L., Attyah, A. M., Cournand, A., Fowell, D. M., Himmelstein, A., Noble, R. P., Remington, J. W., Richards, D. W., Jr., Wheeler, N. C., and Witham, A. C., Comparison of the Fick and dye injection methods of measuring the cardiac output in man. Am. J. Physiol., 1948, 153, 309.

10. Etsten, B. E., and Li, T. H., The determination of cardiac output by the dye dilution method: Modifications, comparison with the Fick method, and application during anesthesia. Anesthesiology, 1954, 15, 217.

11. Li, T. H., Fisher, J. H., and Etsten, B. E., The determination of cardiac output by the dye injection method. Bull. New England M. Center, 1953, 15, 97.

12. Peters, J. P., and Van Slyke, D. A., Quantitative Clinical Chemistry, Volume II, Methods. Baltimore, Williams \& Wilkins Co., 1932.

13. Hamilton, W. F., Moore, J. W., Kinsman, J. M., and Spurling, R. G., Studies on the circulation. IV. Further analysis of the injection method, and of changes in hemodynamics under physiological and pathological conditions. Am. J. Physiol., 1932, 99, 534.

14. Robinson, C. V., Li, T. H., and Etsten, B. E., Unpublished data.

15. Stewart, G. N., The pulmonary circulation time, the quantity of blood in the lungs and the output of the heart. Am. J. Physiol., 1921, 58, 20.
16. Newman, E. V., Merrell, M., Genecin, A., Monge, C., Milnor, W. R., and McKeever, W. P., The dye dilution method for describing the central circulation. An analysis of factors shaping the time-concentration curves. Circulation, 1951, 4, 735.

17. Hamilton, W. F., The physiology of the cardiac output. Circulation, 1953, 8, 527.

18. Etsten, B. E., Rheinlander, H. F., Reynolds, R. N., and $\mathrm{Li}, \mathrm{T}$. H., Mechanism of the reduction of the cardiac output during thiopental and cyclopropane anesthesia. To be published.

19. Stead, E. A., Jr., Warren, J. V., Merrill, A. J., and Brannon, E. S., The cardiac output in male subjects as measured by the technique of right atrial catheterization. Normal values with observations on the effect of anxiety and tilting. J. Clin. Invest., 1945, 24, 326.

20. Cournand, A., Riley, R. L., Breed, E. S., Baldwin, E. deF., and Richards, D. W., Jr., Measurement of cardiac output in man using the technique of catheterization of the right auricle or ventricle. J. Clin. Invest., 1945, 24, 106.

21. Sancetta, S. M., Lynn, R. B., Simeone, F. A., and Scott, R. W., Studies of hemodynamic changes in humans following induction of low and high spinal anesthesia. I. General considerations of the problem. The changes in cardiac output, brachial arterial pressure, peripheral and pulmonary oxygen contents and peripheral blood flows induced by spinal anesthesia in humans not undergoing surgery. Circulation, 1952, 6, 559.

22. Doyle, J. T., Wilson, J. S., Lépine, C., and Warren, J. V., An evaluation of the measurement of the cardiac output and of the so-called pulmonary blood volume by the dye-dilution method. J. Lab. \& Clin. Med., 1953, 41, 29.

23. Li, T. H., Reynolds, R. N., Rheinlander, H. F., and Etsten, B. E., Cardiocirculatory dynamics during thiopental anesthesia in humans. Federation Proc., 1954, 13, 380.

24. Patrick, R. T., and Faulconer, A., Jr., Respiratory studies during anesthesia with ether and pentothal sodium. Anesthesiology, 1952, 13, 252.

25. Price, H. L., Conner, E. H., Elder, J. D., and Dripps, R. D., Effect of sodium thiopental on circulatory response to positive pressure inflation of lung. $\mathrm{J}$. Applied Physiol., 1952, 4, 629.

26. Shackman, R., Graber, G. I., and Redwood, C., Oxygen consumption and anesthesia. Clin. Sc., 1951, 10, 219.

27. Kety, S. S., Woodford, R. B., Harmel, M. H., Freyhan, F. A., Appel, K. E., and Schmidt, C. F., Cerebral blood flow and metabolism in schizophrenia. The effects of barbiturate semi-narcosis, insulin coma and electroshock. Am. J. Psychiat., 1948, 104, 765.

28. Etsten, B., York, G. E., and Himwich, H. E., Pattern of metabolic depression induced with pento- 
thal sodium. Arch. Neurol. \& Psychiat., 1946, 56, 171.

29. Sjöstrand, T., Determinations of changes in the intrathoracic blood volume in man. Acta physiol. Scandinav., 1951, 22, 114.

30. Lagerlöf, H., and Werkö, L., Studies on the circulation of blood in man. VI. The pulmonary capillary venous pressure pulse in man. Scandinav. J. Clin. \& Lab. Invest., 1949, 1, 147.

31. Nylin, G., The relation between the type of the dilution curve and the amount of the residual blood of the heart. Arq. brasil. de cardiol., 1952, 5, 349.

32. Kopelman, H., and Lee, G. de J., The intrathoracic blood volume in mitral stenosis and left ventricular failure. Clin. Sc., 1951, 10, 383.

33. Hausner, E., Essex, H. E., and Mann, F. C., Roentgenologic observations of the spleen of the dog under ether, sodium amytal, pentobarbital sodium and pentothal sodium anesthesia. Am. J. Physiol., 1938, 121, 387.

34. Zweifach, B. W., Hershey, S. G., Rovenstine, E. A., Lee, R. E., and Chambers, R., Anesthetic agents as factors in circulatory reactions induced by hemorrhage. Surgery, 1945, 18, 48.

35. Hershey, S. G., Zweifach, B. W., and Rovenstine, E. A., Effects of depth of anesthesia on behavior of peripheral vascular bed. Anesthesiology, 1953, 14, 245.

36. Shackman, R., Graber, G. I., and Melrose, D. G., The hemodynamics of the surgical patient under general anesthesia. Brit. J. Surg., 1952, 40, 193.

37. Bennett, H. S., Bassett, D. L., and Beecher, H. K., Influence of anesthesia (ether, cyclopropane, sodium evipal) on the circulation under normal and shock conditions. J. Clin. Invest., 1944, 23, 181.

38. Wechsler, R. L., Dripps, R. D., and Kety, S. S., Blood flow and oxygen consumption of the human brain during anesthesia produced by thiopental. Anesthesiology, 1951, 12, 308.

39. Habif, D. V., Papper, E. M., Fitzpatrick, H. F.. Lowrance, P., Smythe, C. McC., and Bradley, S. $\mathrm{E}$., The renal and hepatic blood flow, glomerular filtration rate, and urinary output of electrolytes during cyclopropane, ether, and thiopental anesthesia, operation, and the immediate postoperative period. Surgery, 1951, 30, 241.

40. Papper, E. M., Habif, D. V., and Bradley, S. E., Studies of renal and hepatic function in normal man during thiopental, cyclopropane and high spinal anesthesia. J. Clin. Invest., 1950, 29, 838.

41. Etsten, B. E., and Li, T. H., Effect of carbon dioxide tension upon cardiac output in humans. To be published 